\title{
Experimental Study on Noise Test of small Wind Turbine
}

$$
\text { Bao Daorina }{ }^{1, a} \text {, Shang Wei }{ }^{2, b} \text {, Yao Ming }{ }^{2, c}
$$

${ }^{1,2}$ School of Inner Mongolia University of Technology, huhhot 010051, China

abdrn125@163.com, b1053449142@qq.com

Keywords:Wind Turbine;Noise Test;IEC61400-11;analysis of data

\begin{abstract}
According to the wind turbine noise test method based on IEC61400-11 ,a wind turbine noise rated at $60 \mathrm{KW}$ were tested at"Small and medium-sized wind turbine field test site"in Dongying City, Shandong Province, And the collected data were analyzed, including weighted apparent power level,1/3 frequency doubled range spectrum and pitch.This wind turbine noise emission characteristic of the study found that wind turbine running noise increase with the increase of wind speed is nonlinear, when the background noise has a linear growth with the increase of wind speed, discovered the main ingredients of this wind turbine noise was the broadband noise of $200 \mathrm{~Hz}$ to $2500 \mathrm{~Hz}$, and the abnormal high frequency $6300 \mathrm{~Hz}$ audio was found by the analysis of the pitch.
\end{abstract}

\section{Introduction}

Wind energy, as the most exploitation prospects and the most mature technology of a new renewable energy, has become the focus of attention in the global energy industry.But the vibration and noise of the wind turbine in operation also have impact on a negative impact on the equipment and the environment, physical and mental harm on people, People are becoming more and more aware and concerned.Therefore, in the pursuit of wind power to bring economic and social benefits, generating some negative impact on the environment can not be ignored. While wind turbine noise generated in the equipment itself is very destructive, noise detection equipment status monitoring and fault diagnosis technology in important ways. Therefore, the study of small and medium wind turbine noise testing technique is very necessary.

\section{Calculation of measuring position}

The World Wind Energy Association according to the actual situation of the local promulgated the corresponding wind turbine noise measurement standards, but many of them are based on simplified IEC61400 standards and to.IEC 61400-11 is given a set of wind turbine noise measurement and data processing method.

\subsection{Acoustic measurement position}

Measurement of sound sensor using a microphone, its mounting position as shown in figure 1:

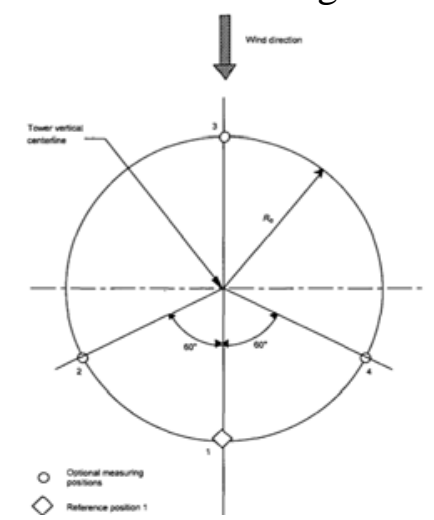

Fig 1. Installation position of microphone

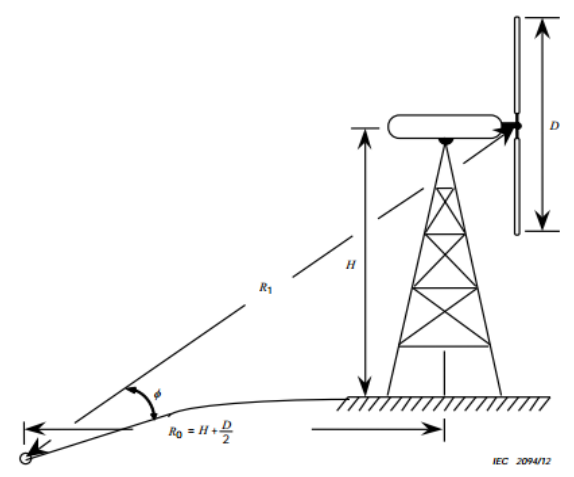

Fig 2. The definition of " $R_{0} 、 R_{1}$ " in the horizontal axis wind turbine 
Figure (1)(2)(3) (4) for the four measurement sites, of which is benchmark to measure the position, it is located in the downwind direction of wind turbine, the (2), (3)and (4) is optional measurement location and used for measuring wind turbine noise directivity.

As figure $2,{ }^{R_{0}}$ set tower of perpendicular to the microphone of the horizontal distance and the $60 \mathrm{~kW}$ horizontal axis wind turbine, $R_{0}$ by (1) determine:

$$
R_{0}=H+\frac{D}{2}
$$

In the formula, $\mathrm{H}$ is the vertical distance from the ground to the center of the wind wheel of the wind turbine, and $\mathrm{D}$ is the diameter of the wind wheel.

\subsection{Measuring position of wind speed and direction}

In this paper, using meteorological tower anemometer direct measurement of wind speed and wind direction, The main vane anemometer and reference anemometer mounted on the meteorological tower,The installation position of the tower as shown in figure 3:

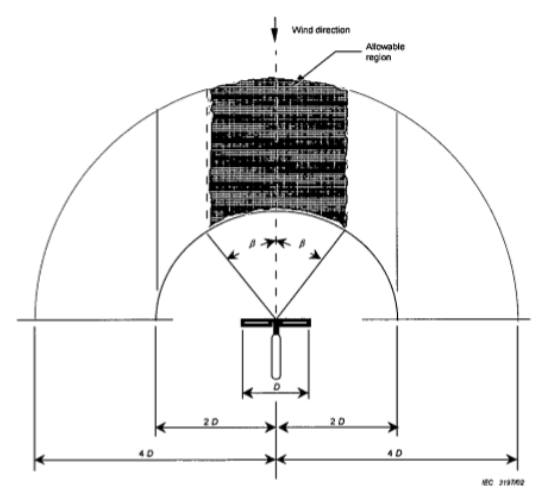

Figure 3. The top view of meteorological tower installation position

Determination of $\beta$ angle:

$$
\beta=\frac{\mathrm{z}-\mathrm{z}_{\text {ref }}}{\mathrm{H}-\mathrm{z}_{\text {ref }}}\left(\beta_{\text {max }}-\beta_{\text {min }}\right)+\beta_{\text {min }}
$$

In the formula: $\mathrm{Z}$ is the height of the wind instrument $(\mathrm{m}) ;{ }^{Z_{\text {ref }}}$ is the reference height, ${ }^{Z_{\text {ref }}}=10 \mathrm{~m}$; $\mathrm{H}$ is the wind turbine wind turbine center or the equator plane height $(\mathrm{m}) ; \beta_{\max }$ is the maximum value of the beta angle, $\beta_{\max }=90$ degrees; $\beta_{\min }$ is the smallest value of the beta angle, $\beta_{\min }=30$;

\subsection{Calculation of standard wind speed}

The measured wind speed is based on the type (3) and (4) the standard wind speed which is converted to the standard atmospheric conditions of wind speed and $10 \mathrm{~m}$ height and ground surface roughness under $0.05 \mathrm{~m}$ conditions[4,5].

$$
V_{z n}=V_{z n}\left[\frac{p}{T_{k}} \cdot \frac{T_{r e f}}{p_{r e f}}\right]^{1 / 3}
$$

In the formula: $V_{z m}$ is the wind speed of the measured wind speed, that is, measured wind speed, $\mathrm{m} / \mathrm{s} \quad p$ is the measured atmospheric pressure, $\mathrm{kPa} ; T_{k}$ is the measured air temperature, $\mathrm{K} ; T_{\text {ref }}$ is the reference air temperature, $T_{0}=288 \mathrm{~K} ; p_{\text {ref }}$ for reference atmospheric pressure, $P_{0}=101.325 \mathrm{kPa}$.

$$
V_{s}=V_{z n}\left(\frac{\ln \left(\frac{z_{\text {ref }}}{z_{\text {oref }}}\right) \ln \left(\frac{H}{z_{0}}\right)}{\ln \left(\frac{H}{z_{\text {oref }}}\right) \ln \left(\frac{z}{z_{0}}\right)}\right)
$$

In the formula: $V_{z n}$ is the wind speed of standard atmospheric conditions, $\mathrm{m} / \mathrm{s} ; \quad z_{0 r e f}$ is reference roughness length, $0.05 \mathrm{~m} .{ }^{z_{0}}$ is the terrain roughness length, $\mathrm{m},{ }_{r \text { ref }}$ is the reference height $10 \mathrm{~m} ; \mathrm{Z}$ is the wind tower anemometer heights, $\mathrm{m} ; H$ is the height of the center of the wind wheel, $\mathrm{M}$; 


\section{Experimental analysis}

The 61400-11 IEC international standard requirements for wind turbine noise to do three aspects of the analysis, that is, the standard wind speed $(6,7,8,9,10 \mathrm{~m} / \mathrm{s})$ of the A sound power level, $1 / 3$ times octave spectrum and tone analysis.In this paper, according to the IEC61400-11 standard for a certain type of $60 \mathrm{KW}$ wind turbine noise test data acquisition. The system took a total of 1379 noise data acquisition, which run the noise 920, the background noise 459.The wind speed is measured by the formula (3) and (4)of the wind speed to get the standard wind speed. Analysis 3 to $12 \mathrm{~m} / \mathrm{s}$ standard wind speed under the apparent sound power level is as shown in table 1:

Table 1. Calculation results of sound power level

\begin{tabular}{ccccc}
$\begin{array}{l}\text { Standardized wind } \\
\text { speed }(\mathrm{m} / \mathrm{s})\end{array}$ & $\begin{array}{l}\text { Sound pressure } \\
\text { level of } \\
\text { operating } \\
\text { noise(dB) }\end{array}$ & $\begin{array}{l}\text { Sound pressure } \\
\text { level of } \\
\text { background } \\
\text { noise(dB) }\end{array}$ & $\begin{array}{l}\text { Corrected value } \\
\left(L_{\text {Aeq, }, k}\right)\end{array}$ & $\begin{array}{l}\text { Acoustical power } \\
\left(L_{\text {wa,k }}\right)\end{array}$ \\
\hline 3 & 50.83 & 38.25 & 50.58 & 91.34 \\
4 & 52.21 & 36.3 & 52.1 & 92.86 \\
5 & 54.22 & 39.48 & 54.08 & 94.83 \\
6 & 56.61 & 40.85 & 56.5 & 97.25 \\
7 & 57.29 & 42.51 & 57.14 & 97.9 \\
8 & 58.03 & 45.62 & 57.77 & 98.53 \\
9 & 58.28 & 50.16 & 57.56 & 98.31 \\
10 & 58.74 & 51.41 & 57.85 & 98.61 \\
11 & 59.02 & 52.9 & 57.8 & 98.56 \\
12 & 59.53 & 53.03 & 58.43 & 99.18 \\
\hline
\end{tabular}

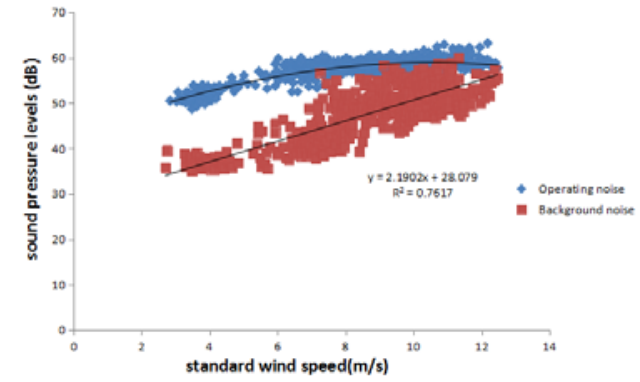

Figure 4. Running and stopping noise pressure levels of A weighted

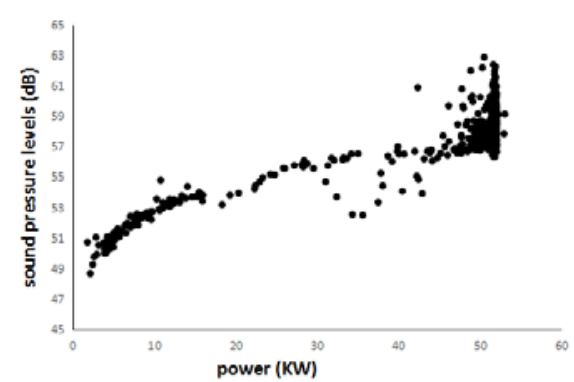

Fig 5. Relationship between power and of sound sound pressure level

Table 2. Results of $1 / 3$ times octave spectrum analysis under the integer wind speed $8 \mathrm{~m} / \mathrm{s}$

\begin{tabular}{ccccccccc}
\hline $\begin{array}{l}\text { Center } \\
\text { frequency } \\
(\mathrm{Hz})\end{array}$ & $\begin{array}{c}\text { group } \\
(\mathrm{dB})\end{array}$ & $\begin{array}{l}2 \text { group } \\
(\mathrm{dB})\end{array}$ & $\begin{array}{c}3 \text { group } \\
(\mathrm{dB})\end{array}$ & $\begin{array}{c}\text { Backgroud } \\
\text { noise }(\mathrm{dB})\end{array}$ & $\begin{array}{c}\text { Corrected } \\
\text { value }(\mathrm{dB})\end{array}$ & $\begin{array}{c}\text { Acoustical } \\
(\mathrm{dB})\end{array}$ & UA $(\mathrm{dB})$ & UC $(\mathrm{dB})$ \\
\hline 200 & 37.39 & 39.57 & 40.58 & 35.21 & 38.07 & 78.83 & 1.65 & 2.76 \\
250 & 41.37 & 43.87 & 45.46 & 36.35 & 43.04 & 83.79 & 2.1 & 2.22 \\
315 & 42.63 & 44.34 & 47.35 & 36.55 & 44.58 & 85.34 & 2.45 & 2.55 \\
400 & 43.7 & 45.17 & 49.24 & 36.01 & 46.31 & 87.07 & 2.98 & 3.06 \\
500 & 44.4 & 46.19 & 48.81 & 39.3 & 46 & 86.76 & 2.27 & 2.67 \\
630 & 46.41 & 47.31 & 50.84 & 38.93 & 48.14 & 88.9 & 2.4 & 2.56 \\
800 & 46.08 & 46.85 & 49.8 & 37.94 & 47.42 & 88.18 & 2 & 2.13 \\
1000 & 46.68 & 47.01 & 49.95 & 38.92 & 47.59 & 88.35 & 1.83 & 2.07 \\
1250 & 46.79 & 46.99 & 48.18 & 37.17 & 46.93 & 87.68 & 0.75 & 1.03 \\
1600 & 46.72 & 46.62 & 46.49 & 35.91 & 46.23 & 86.98 & 0.11 & 0.71 \\
2000 & 45.21 & 45.46 & 43.2 & 33.56 & 44.39 & 85.15 & 1.25 & 1.45 \\
2500 & 41.37 & 42.15 & 43.01 & 33.02 & 41.67 & 82.43 & 0.82 \\
\hline
\end{tabular}

And the fitting curves and equations of the background noise and the running time of the corresponding standard wind speed can be obtained by the calculation and the regression analysis.

As shown in Figure 4, we can know that the background noise is linear correlation with the standard wind speed, the fitting equation is $y=2.1902 x+28.079$, the regression analysis results for its 
correlation coefficient $\mathrm{R}=0.872$, the coefficient of

determination R2=0.761Running noise and standardized wind speed showed a nonlinear relationship, .the reason is that the noise generation mechanism of wind turbine is complex, and the noise is not only related to wind conditions and operation characteristics. And when the wind speed is low, the main component of background noise is not the wind turbine operation, Wind power at this time unit itself produced by mechanical noise is running total noise of main component. When the wind speed

is larger, the background noise is the main component of the noise of wind turbine.

Analysis of output power and the sound pressure level of the correspondence between the test obtained as shown in Figure 5 scattered point diagram. Before it can be seen from the figure, in the unit to achieve the rated power, sound pressure level increases with the increase of output power. Is about to reach the rated power, the sound pressure level appear fluctuation, the reasons for this situation is unit is about to reach the rated power, pitch regulated power output, the variable propeller mechanism and the control system sends out the noise fluctuations.

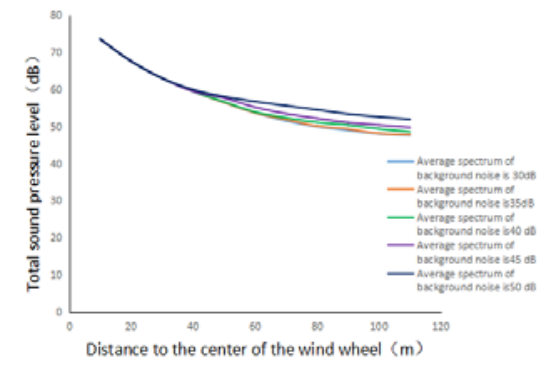

Figure 6. The total noise and the wind distance function diagram

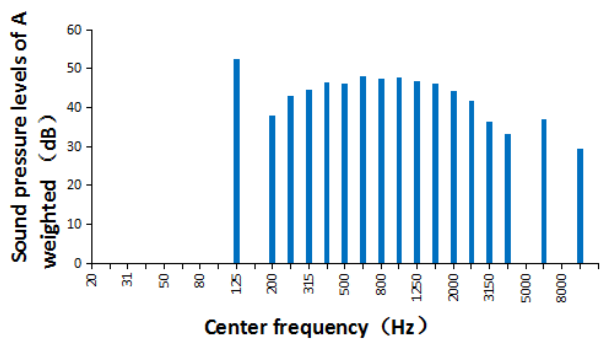

Fig. 7 1/3 times octave spectrum after wheel center background noise correction

Figure 6 shows the under different background noise to the graph of the distance of the center of the wind wheel and of the total sound pressure level, this figure reflects the size of the noise sound pressure level at different positions of the radiation wind turbine.

As can be seen from the figure, the noise of the wind turbine is much smaller. This can help the user to choose the appropriate installation location of the wind turbine.This can help users to choose suitable wind turbine installation positon.It can be seen from the figure, in front of the distance to the wind wheel center $54 \mathrm{~m}$, the total sound pressure level decreased rapidly with increasing the distance,The leading role of the noise and the background noise of the unit basically no contribution to the overall sound pressure level; after, the downward trend slowed, background noise is increased.

Such as $1 / 3$ times octave spectrum analysis results of integer wind speed $8 \mathrm{~m} / \mathrm{s}$, as is shown in Figure $7,1 / 3$ times octave spectrum after background noise correction. IEC require wind turbine noise of $1 / 3$ times octave spectrum by the average energy of at least three spectral bands identified, at least as measured center frequency $50 \mathrm{~Hz} \sim 10 \mathrm{kHz} 1 / 3$ octave belt. Table 2 shows the 1/3 octave analysis results of $8 \mathrm{~m} / \mathrm{s}$ wind speed under, From $200 \mathrm{~Hz}$ to $2500 \mathrm{~Hz}$ in a wide frequency range, the spectral line changes smoothly, and its value is higher, therefore, the frequency range of a wide band noise is the main component of wind noise.

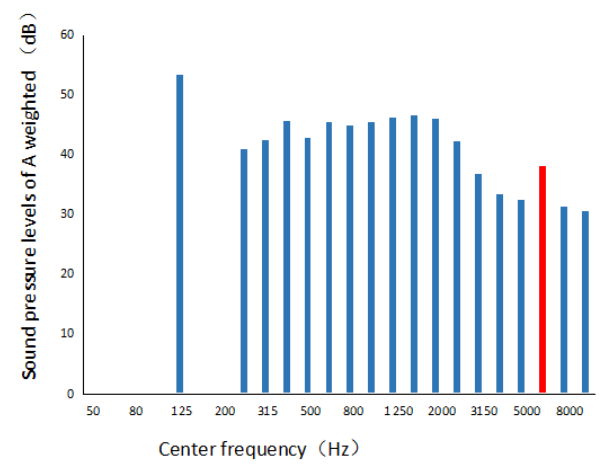

Fig 8. 1/3 times octave spectrum after background noise correction 
Table 3. The results of the tone analysis of $8 \mathrm{~m} / \mathrm{s}$ under the wind speed

\begin{tabular}{|c|c|c|c|c|c|c|c|c|}
\hline $\begin{array}{l}\text { Center } \\
\text { frequency } \\
(\mathrm{Hz})\end{array}$ & $\begin{array}{l}\text { Operating } \\
\text { noise } 1 \\
(\mathrm{~dB})\end{array}$ & $\begin{array}{l}\text { Operating } \\
\text { noise } 2 \\
(\mathrm{~dB})\end{array}$ & $\begin{array}{c}\text { Operating } \\
\text { noise } 3 \\
(\mathrm{~dB})\end{array}$ & $\begin{array}{c}\text { Background } \\
\text { noise } 1 \\
\text { (dB })\end{array}$ & $\begin{array}{c}\text { Background } \\
\text { noise } 2 \\
\text { (dB) }\end{array}$ & $\begin{array}{c}\text { Background } \\
\text { noise } 3 \\
\text { (dB ) }\end{array}$ & $\begin{array}{l}\text { Average } \\
\text { spectrum } \\
\text { of } \\
\text { operating } \\
\text { noise } \\
\text { (dB) }\end{array}$ & $\begin{array}{l}\text { Corrected } \\
\text { of } \\
\text { background } \\
\text { (dB) }\end{array}$ \\
\hline 125 & 52.56 & 54.15 & 53.5 & 40.6 & 36.58 & 29.18 & 53.45 & 53.34 \\
\hline 160 & 36.25 & 36.72 & 37.24 & 35.23 & 36.87 & 30.5 & 36.75 & * \\
\hline 200 & 37.42 & 37.79 & 37.81 & 34.91 & 36.94 & 33.53 & 37.68 & * \\
\hline 250 & 42.09 & 42.37 & 42.43 & 36.48 & 37.55 & 34.89 & 42.3 & 41 \\
\hline 315 & 40.78 & 45.98 & 41.51 & 37.17 & 37.26 & 35.2 & 43.41 & 42.38 \\
\hline 400 & 41.7 & 49.62 & 42.42 & 36.31 & 35.84 & 36.31 & 46.16 & 45.7 \\
\hline 500 & 42.39 & 46.12 & 43.01 & 41.77 & 35.62 & 38.22 & 44.16 & 42.86 \\
\hline 630 & 44.89 & 47.76 & 45.47 & 41.52 & 36.03 & 36.95 & 46.22 & 45.34 \\
\hline 800 & 43.83 & 47.7 & 44.53 & 40 & 35.98 & 36.7 & 45.7 & 44.9 \\
\hline 1000 & 44.88 & 47.82 & 45.39 & 41.57 & 35.97 & 36.63 & 46.23 & 45.35 \\
\hline 1250 & 45.87 & 47.63 & 46.16 & 38.79 & 35.47 & 36.33 & 46.62 & 46.11 \\
\hline 1600 & 46.65 & 47.11 & 46.81 & 37.59 & 33.95 & 35.34 & 46.86 & 46.5 \\
\hline 2000 & 46.51 & 45.88 & 46.29 & 35.25 & 30.06 & 33.94 & 46.24 & 45.99 \\
\hline 2500 & 42.7 & 42.79 & 42.58 & 34.69 & 27.82 & 33.92 & 42.69 & 42.2 \\
\hline 3150 & 36.66 & 39.2 & 37.28 & 31.87 & 27.09 & 33.04 & 37.85 & 36.76 \\
\hline 4000 & 33.78 & 35.57 & 34.49 & 30.35 & 24.15 & 32.42 & 34.68 & 33.38 \\
\hline 5000 & 32.91 & 34.53 & 33.76 & 30 & 23.71 & 31.61 & 33.78 & 32.48 \\
\hline 6300 & 38.19 & 38.91 & 38.37 & 29.13 & 24.32 & 30.56 & 38.5 & 38.02 \\
\hline 8000 & 32.16 & 32.99 & 32.57 & 27.71 & 24.33 & 29.59 & 32.59 & 31.29 \\
\hline
\end{tabular}

Such as figure 8 such as 1/3 times octave spectrum after background noise correction, the calculated numerical results show, the peak spectral line is higher than that of the two sides of the spectrum line more than $5 \mathrm{~dB}$, as shown in Table 3 gives some dates $1 / 3$ times octave spectrum after background noise correction. Therefore, according to the standard the IOS 1996-2:2007 do provisions of the peak spectral line for tonal noise.Taking into account of the aerodynamic noise frequency and wind speed and wind wheel speed related. And $6300 \mathrm{~Hz}$ peak spectral line exists in all wind speed, so it does not belong to the aerodynamic noise. Such a high frequency noise generated according to the experience in general mechanical components will not, in and set manufacturer technical staff to communicate that the noise of the $6300 \mathrm{~Hz}$ converter set issued, which belongs to a kind of electromagnetic noise.

\section{Conclusion}

In this paper, through the analysis of the noise test data of a certain type of 600KW wind power generator, the characteristics of acoustic emission of wind generator are obtained, which can be used as reference for the researchers who are engaged in the noise testing of small and medium sized wind power generation units.Through the experiment it was found that (1)with the wind speed increases ,the wind turbine noise and background noise increases, and the background noise is approximate linear correlation trend growth, the correlation coefficient of 0.872 . When the wind speed is low, the background noise is nonlinear. (2) when the wind speed is low, the wind speed is low, the noise of the wind power generator is the main component of the total noise during operation. When the wind speed is heavy, the background noise is the main component of the total noise of the wind power generator.(3) The analysis of total upgrade atlas found that before the distance to the wind wheel center $54 \mathrm{~m}$, the overall sound pressure level drops rapidly with the increase of the distance, when the wind turbine noise play main role. Background noise from the main role after a slow decline.(4) $200 \mathrm{~Hz}$ to $2500 \mathrm{~Hz}$ frequency range of a wide range of broadband noise is the main component of wind noise.(5) Spectrum analysis found that the frequency from $2000 \mathrm{~Hz}$ to $6300 \mathrm{~Hz}$ broadband noise is the main component of the noise of the unit. The $6300 \mathrm{~Hz}$ 
tone noise is the generator of the electromagnetic noise.

\section{References}

[1] Lei Jun,Zhang Hairong.Test research on vibrations and noise of wind turbine and noise reduction method[J].Northern Environmental,2011，23（12）101-104.

[2] Xin Haisheng,Tian De,et al.Comparative experiment and study on the noise from different types wind turbine generator[J].Journal of Inner Mongolia Agricultural University, 2013,45 (2) : 187-190.

[3] Yao Xinjia. wind power generation test technology[M].Beijing Electronic industry press,2011.

[4] Wu Honggang,Qin Shuren.Research on vibration detection and noise evaluation of wind turibines[D].Chongqing University,2010.

[5] Sun Lei,qin Shuren,bo lin.Acoustic emission assessment of wind turbines based on IEC standard[J].2008,34(1): 5-9.

[6] IEC61400-11-2012,Wind turbines-Part11:Acoustic noise measurement techniques(2012)[S].

[7] ISO1996-2:Acoustic-Description, measurement and assessment of environmental noise-Part 2: Determination of environmental noise levels(2007)[S]. 